\title{
Targeting glutamine metabolism sensitizes pancreatic cancer to PARP-driven metabolic catastrophe induced by ß-lapachone
}

Gaurab Chakrabarti ${ }^{1,2}$, Zachary R. Moore ${ }^{1,2}$, Xiuquan Luo ${ }^{1,2}$, Mariya Ilcheva², Aktar Ali ${ }^{3}$, Mahesh Padanad ${ }^{4}$, Yunyun Zhou ${ }^{5}$, Yang Xie ${ }^{5}$, Sandeep Burma ${ }^{2}$, Pier P. Scaglioni ${ }^{4}$, Lewis C. Cantley ${ }^{6}$, Ralph J. DeBerardinis ${ }^{7}$, Alec C. Kimmelman ${ }^{8}$, Costas A. Lyssiotis ${ }^{9,10^{*}}$ and David A. Boothman ${ }^{1,2^{*}}$

\begin{abstract}
Background: Pancreatic ductal adenocarcinomas (PDA) activate a glutamine-dependent pathway of cytosolic nicotinamide adenine dinucleotide phosphate (NADPH) production to maintain redox homeostasis and support proliferation. Enzymes involved in this pathway (GLS1 (mitochondrial glutaminase 1), GOT1 (cytoplasmic glutamate oxaloacetate transaminase 1), and GOT2 (mitochondrial glutamate oxaloacetate transaminase 2)) are highly upregulated in PDA, and among these, inhibitors of GLS1 were recently deployed in clinical trials to target anabolic glutamine metabolism. However, single-agent inhibition of this pathway is cytostatic and unlikely to provide durable benefit in controlling advanced disease.

Results: Here, we report that reducing NADPH pools by genetically or pharmacologically (bis-2-(5-phenylacetamido-1,2, 4-thiadiazol-2-yl)ethyl sulfide (BPTES) or (B-839) inhibiting glutamine metabolism in mutant Kirsten rat sarcoma viral oncogene homolog (KRAS) PDA sensitizes cell lines and tumors to ß-lapachone (ß-lap, clinical form ARQ761). ß-Lap is an NADPH:quinone oxidoreductase (NQO1)-bioactivatable drug that leads to NADPH depletion through high levels of reactive oxygen species (ROS) from the futile redox cycling of the drug and subsequently nicotinamide adenine dinucleotide (NAD)+ depletion through poly(ADP ribose) polymerase (PARP) hyperactivation. NQO1 expression is highly activated by mutant KRAS signaling. As such, B-lap treatment concurrent with inhibition of glutamine metabolism in mutant KRAS, NQO1 overexpressing PDA leads to massive redox imbalance, extensive DNA damage, rapid PARP-mediated NAD+ consumption, and PDA cell death—features not observed in NQO1-low, wild-type KRAS expressing cells.

Conclusions: This treatment strategy illustrates proof of principle that simultaneously decreasing glutamine metabolism-dependent tumor anti-oxidant defenses and inducing supra-physiological ROS formation are tumoricidal and that this rationally designed combination strategy lowers the required doses of both agents in vitro and in vivo. The non-overlapping specificities of GLS1 inhibitors and B-lap for PDA tumors afford high tumor selectivity, while sparing normal tissue.
\end{abstract}

Keywords: Metabolic cancer therapy, Glutamine metabolism, Transamination, NQO1-bioactivated drugs

\footnotetext{
* Correspondence: clyssiot@med.umich.edu; David.Boothman@

UTSouthwestern.edu

${ }^{9}$ Department of Molecular and Integrative Physiology, University of Michigan,

Ann Arbor, Ml 48109, USA

'Department of Pharmacology, University of Texas Southwestern Medical

Center, 6001 Forest Park Drive, Dallas 75390-8807TX, USA

Full list of author information is available at the end of the article
}

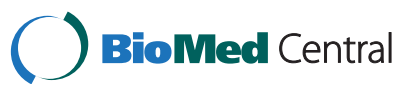

(c) 2015 Chakrabarti et al. Open Access This article is distributed under the terms of the Creative Commons Attribution 4.0 International License (http://creativecommons.org/licenses/by/4.0/), which permits unrestricted use, distribution, and reproduction in any medium, provided you give appropriate credit to the original author(s) and the source, provide a link to the Creative Commons license, and indicate if changes were made. The Creative Commons Public Domain Dedication waiver (http://creativecommons.org/publicdomain/zero/1.0/) applies to the data made available in this article, unless otherwise stated. 


\section{Background}

Pancreatic ductal adenocarcinoma (PDA) is a recalcitrant cancer in which patients have $<6 \% 5$-year survival rates. Mortality from this disease is predicted to be the second leading cause of cancer-related death by 2020 [1]. PDAs are highly resistant to conventional chemotherapies [1], and activating mutations in Kirsten rat sarcoma viral oncogene homolog $(K R A S)$ are present in $>95 \%$ of all cases [2]. Despite significant effort from the pharmaceutical industry over the past 30 years, KRAS has proven to be a challenging drug target [3]. An emerging therapeutic approach is to target alterations in PDA metabolism driven by mutant KRAS [2, 4-6]. For example, PDA cells generate the bulk of the ribose used for de novo nucleotide biosynthesis through the non-oxidative arm of the pentose phosphate pathway [7]. This KRAS-driven reprogramming of glucose metabolism bypasses the nicotinamide adenine dinucleotide phosphate (NADPH)-generating oxidative arm. To compensate for this rewiring, PDAs utilize glutamine through a GLS1 (mitochondrial glutaminase)-, GOT2 (mitochondrial glutamate oxaloacetate transaminase 2)-, and GOT1 (cytoplasmic glutamate oxaloacetate transaminase 1)-dependent pathway to support cellular redox balance in the face of rapid proliferation and growth (Fig. 1a) [2, 8, 9]. This is in contrast to the canonical metabolism of glutamine-derived glutamate through GLUD1 (glutamate dehydrogenase 1) to supply carbon backbone to the TCA cycle. Genetic inhibition of enzymes in this pathway is profoundly growth inhibitory in PDA but does not result in the induction of a cytotoxic response. These results suggest that a means to induce redox balance in PDA, concurrent with inhibition of this KRAS-dependent Gln metabolism pathway, may provide a means to induce tumor-selective killing.

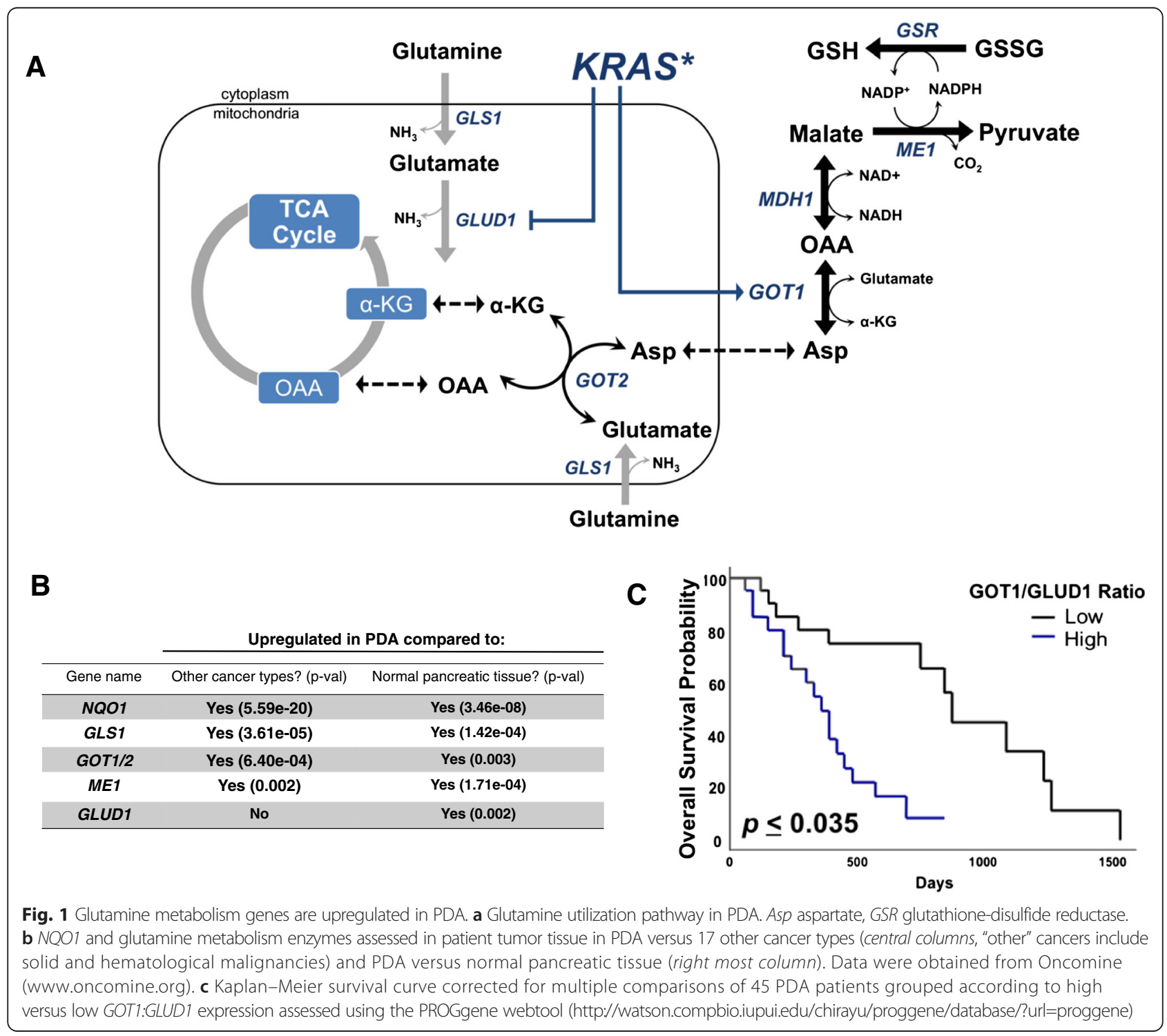


In an attempt to leverage increased tumor-cell reliance on glutamine, small molecule inhibitors of GLS1 were developed (e.g., bis-2-(5-phenylacetamido-1,2,4-thiadiazol-2-yl)ethyl sulfide (BPTES), CB-839, compound 968) [10-12]. GLS1 catalyzes the first step in the PDA glutamine metabolism pathway, converting glutamine to glutamate (Fig. 1a) [8]. As such, GLS1 inhibition in PDA cells in culture leads to a block in glutamine metabolism but, as with the genetic approaches above, lacks cytotoxicity. Moreover, while GLS1 inhibitors are potent inhibitors of cell proliferation in cell culture models, they have relatively minor effects on tumor growth in pre-clinical cancer models as single agents [13-17].

To increase the specificity and efficacy of GLS1 inhibition in PDA, we combined BPTES or CB-839 with ß-lapachone (ß-lap), a targeted cancer therapeutic that causes tumorselective reactive oxygen species (ROS) formation in an NADPH:quinone oxidoreductase 1 (NQO1)-specific manner [18]. NQO1 is highly expressed in many types of cancer, including PDA. In fact, elevated NQO1 expression ( $\geq$ tenfold) has been observed in $\sim 90 \%$ of PDA patient specimens, making PDA an especially appealing target for therapy using NQO1-bioactivatable drugs, such as $ß$-lap [18-21].

ß-Lap is a substrate for two-electron oxidoreduction by NQO1, an inducible phase II quinone-detoxifying enzyme. The resulting hydroquinone form of $\beta$-lap is highly unstable and spontaneously reacts with oxygen to revert back to the parent compound, generating two moles of superoxide per mole $\mathrm{NAD}(\mathrm{P}) \mathrm{H}$ used in the process [18]. This leads to a futile cycle that occurs rapidly in NQO1-overexpressing cells resulting in massive ROS formation, oxidative DNA damage, and $\mathrm{H}_{2} \mathrm{O}_{2}$-mediated DNA single-strand breaks (Additional file 1: Figure S1A). In an attempt to repair this damage, poly(ADP ribose) polymerase $(P A R P)$ becomes hyperactivated, generating extensive free branched poly(ADP ribose) (PAR) polymer levels. The hyperactivated PARP substantially depletes intracellular nicotinamide adenine dinucleotide (NAD)+ and adenosine triphosphate (ATP) pools and ultimately overwhelms the ability of the DNA repair machinery to repair ß-lap-induced DNA lesions.

The therapeutic window provided by NQO1 expression (and thus NQO1-mediated bioactivation of $B$-lap) has advanced B-lap to phase I and Ib clinical trials (ARQ761) [22]. Unfortunately, dose-limiting methemoglobinemia caused by nonspecific ROS generation at high $\beta$-lap doses somewhat limits the efficacy of $ß$-lap as monotherapy [22]. Strategies for increasing cancer cell cytotoxicity, while maintaining NQO1 specificity, could further enhance efficacy of $ß$-lap for therapy against PDAs.

ß-Lap and GLS1 inhibition have distinct but highly complementary mechanisms of action. ß-Lap induces tumor-selective ROS generation specifically in PDA cells that express high levels of NQO1. GLS1 inhibition primes PDA cancer cells for death by lowering anti-oxidant pools derived from glutamine, sensitizing the cell to ROS damage. Here we show, using an in vivo pre-clinical model of PDA, that the increased dependence of PDA cells on glutamine is specifically targeted by exposure to both drugs. The use of ß-lap with GLS1 inhibitors results in synergistic NQO1- and PARP-dependent cancer cell death, allowing use of lower doses and shorter treatment times for both agents.

\section{Results}

\section{Glutamine metabolism genes are upregulated in PDA}

Enzymes utilized for glutamine metabolism in PDA, GLS1, GOT1/2, and malic enzyme 1 (ME1) (see pathway, Fig. 1a), in addition to NQO1, were significantly upregulated in PDA compared to 17 other cancers when assessed using the Oncomine webtool (Fig. 1b) [8]. This was apparent in both cell lines and tumor samples. In contrast, GLUD1, which diverts glutamine carbon away from the GOT2-GOT1-ME1 pathway into an alternate metabolic pathway, was not upregulated in PDA relative to other cancer types (Fig. 1a). Additionally, glutamine metabolic enzymes, NQO1, and GLUD1 were found to be significantly upregulated in PDA relative to normal pancreatic tissue (Fig. 1a). To determine the clinical relevance associated with the PDA glutamine metabolic pathway relative to other enzymes involved in glutamine metabolism, we evaluated the association of individual gene expression levels with overall survival in the data set that contained clinical follow-up information [23]. Kaplan-Meier analysis did not show a significant difference in outcome when patients were separated into high and low expression levels of the genes of interest (data not shown). However, when GOT1 and GOT2 were normalized to GLUD1 expression, we found that a high GOT1 to GLUD1 or GOT2 to GLUD1 ratio was significantly associated with poor outcome (Fig. 1c, Additional file 1: Figure S1B). These data suggest that gene expression of GOT isoforms are generally elevated in PDA and have prognostic and functional significance relative to GLUD1 expression.

\section{Inhibiting glutamine metabolism sensitizes PDA to B-lap} Given the reliance of PDA on glutamine metabolism for redox balance, we hypothesized that glutamine deprivation of NQO1-overexpressing PDA cells would sensitize them to ß-lap exposure by lowering anti-oxidant defenses and increasing NQO1-induced ROS damage. MiaPaCa2 cells were grown in Gln-free or Gln-containing $(2 \mathrm{mM})$ media for $16 \mathrm{~h}$ and then exposed to ß-lap for $2 \mathrm{~h}$. Short-term Gln deprivation did not significantly alter clonogenic survival on its own (Fig. 2a) but did sensitize MiaPaCa2 cells to ß-lap at sub-lethal and higher doses of the drug (Fig. 2a). To confirm these results, we repeated this 


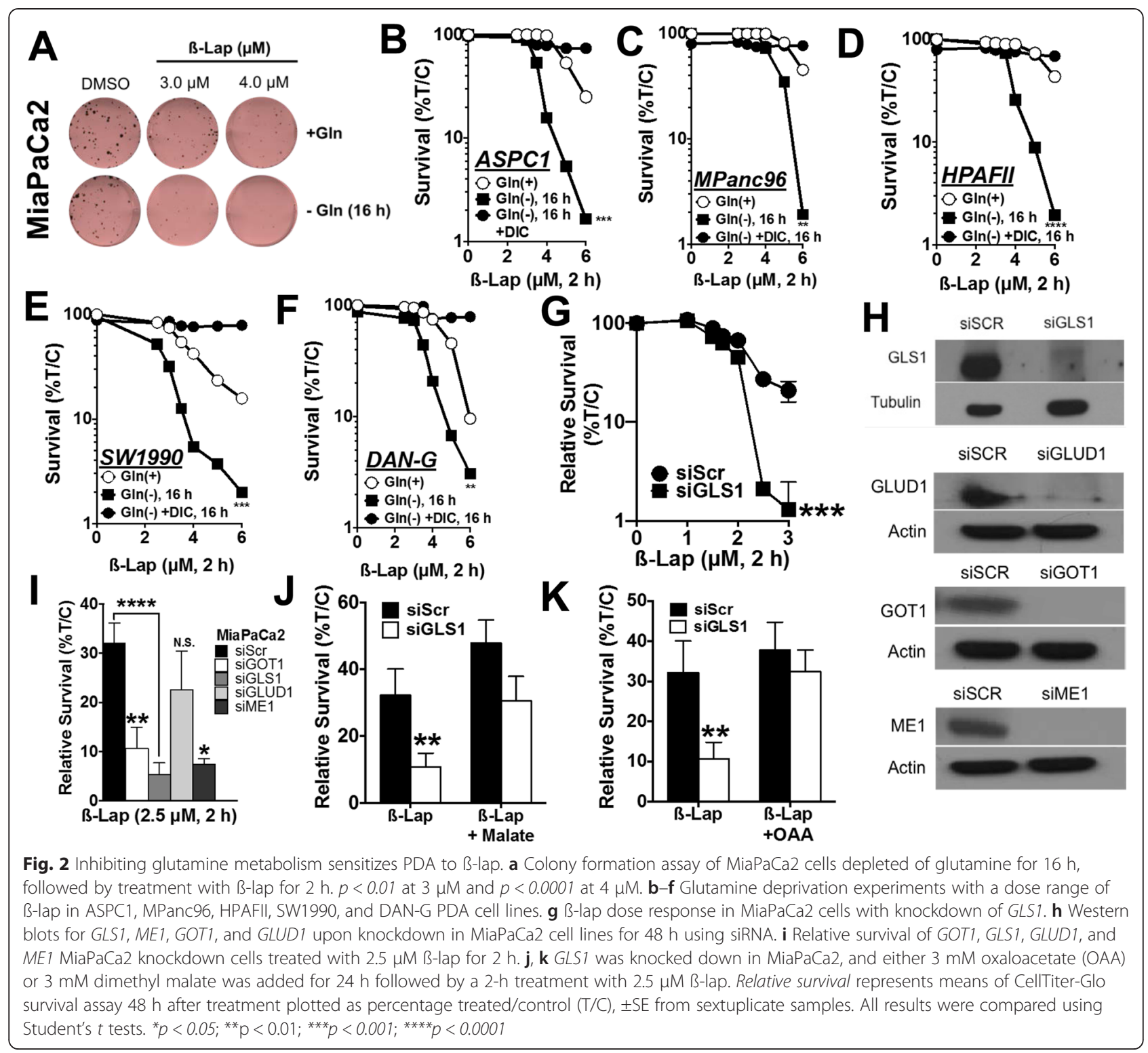

experiment in five other PDA cell lines: ASPC1, MPanc96, HPAFII, SW1990, and DAN-G (Fig. 2b-f). Additionally, we demonstrated that the observed cytotoxicity was NQO1-dependent, and as addition of the potent NQO1 inhibitor, dicoumarol (DIC), spared cells from lethality (Fig. 2b-f) [24-26].

Next, RNAi-mediated knockdown of glutamine metabolism enzymes revealed that GLS1, GOT1, and ME1 dramatically sensitized MiaPaCa2 and ASPC1 PDA cell lines to $ß$-lap, relative to non-targeting control (scramble small interfering RNA (siScr)) (Fig. 2g-k). Consistent with the mechanism by which glutamine is metabolized in PDA to maintain redox balance [8], knockdown of GLUD1 had no effect on ß-lap sensitivity (Fig. 2i). Sensitization of ß-laptreated MiaPaCa2 cells by GLS1 knockdown was rescued by replenishing metabolic substrates of the glutamine metabolism pathway that are downstream of the GLS1 reaction, namely, oxaloacetate (OAA) or cell-permeable dimethyl malate (Fig. 2j, k). These data indicate that PDA cells have an increased reliance on glutamine to generate NADPH (Fig. 1a) in the presence of $ß$-lap-induced ROS stress.

\section{GLS1 inhibition by BPTES sensitizes PDA to ß-lap in an NQO1-dependent manner}

To pharmacologically replicate the $ß$-lap sensitization to inhibition of Gln metabolism, MiaPaCa2 cells were treated with a sub-lethal dose of the mitochondrial GLS1 inhibitor, BPTES (500 nM, 48 h, Additional file 2: Figure S2) and then exposed to various doses of $ß$-lap for $2 \mathrm{~h}$, with or 
without DIC (Fig. 3a). BPTES pre-treatment in combination with ß-lap significantly reduced clonogenic survival versus 3 -lap alone, while addition of DIC spared the lethality (Fig. 3a). To confirm that our results were due to inhibition of the glutamine-dependent transamination pathway and not the alternative glutamine metabolism pathway through GLUD1, we pre-treated MiaPaCa2 cells with epigallocatechin gallate (EGCG), an inhibitor of GLUD1 [27] for $48 \mathrm{~h}$ and then exposed them to B-lap. Consistent with our RNAi results (Fig. 2i), we found that GLUD1 inhibition by EGCG had no effect on ß-lap sensitivity (Fig. 3b). Furthermore, normal human IMR-90 embryonic lung fibroblasts, which have low NQO1 levels [26] were not affected by ß-lap, with or without BPTES treatments (Fig. 3c). Replenishing the NADPH-producing transamination pathway with the addition of OAA or dimethyl malate, metabolites downstream of GLS1, rescued BPTES-dependent hypersensitivity to $ß$-lap in MiaPaCa2, ASPC1, and HPAFII PDA cells (Fig. 3d).
Mutant KRAS signaling drives NQO1 expression and glutamine dependence $[7,8,28,29]$, thus we sought to assess the generality of mutant KRAS expression on the effects of BPTES and B-lap. BPTES pre-treatment sensitized a variety of $N Q O 1$-expressing KRAS mutant cancer cell lines to $ß$-lap, including lung, triple-negative breast, and additional PDA cell lines. In contrast, NQO1-deficient KRAS mutant lines remained resistant to ß-lap, whether or not BPTES was added (Fig. 3e). In addition, pre-treatment with $500 \mathrm{nM}$ BPTES for $48 \mathrm{~h}$ did not increase the sensitivities of ß-lap-responsive, NQO1expressing KRAS wild-type lung, breast, or pancreatic cancer cell lines, consistent with reported literature $[8,30]$. Collectively, these results illustrate that in order for this targeted combination of agents to be effective, sensitive cells must exhibit both mutant KRAS-driven Gln dependence and NQO1 expression. The latter is a feature of most, but not all, mutant $K R A S$-transformed cancer types.

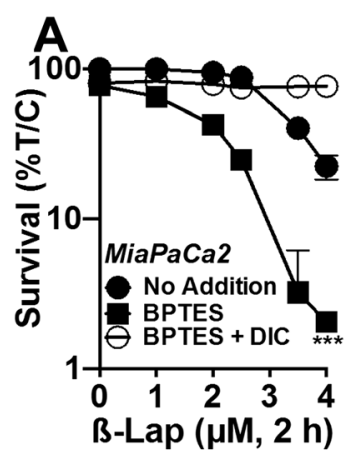

D

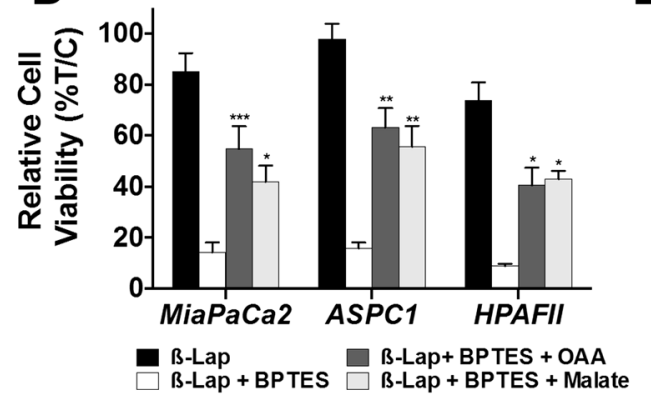

B

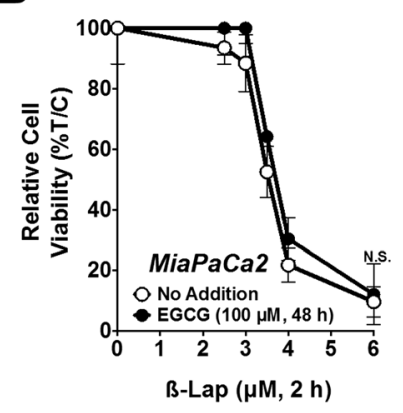

C

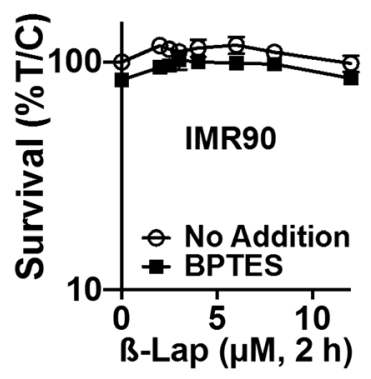

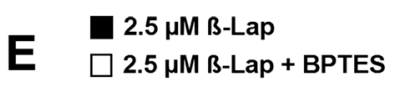

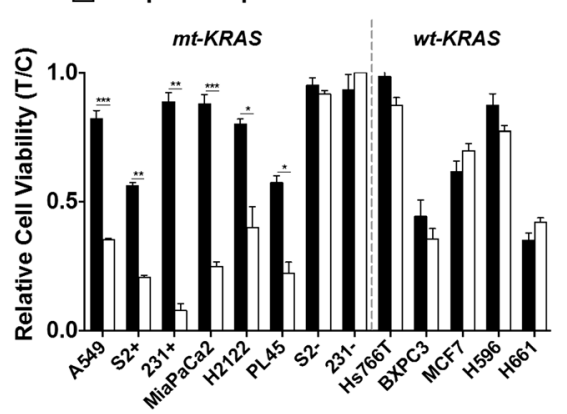

Fig. 3 GLS1 inhibition by BPTES sensitizes PDA to ß-lap in an NQO1-dependent manner. a Clonogenic survival assay of MiaPaCa2 pre-treated $\pm 500 \mathrm{nM}$ BPTES for $48 \mathrm{~h}$ followed by the addition of B-lap $\pm 50 \mu \mathrm{M}$ for $2 \mathrm{~h}$. Data represent survival means \pm SE from quadruplicate samples. b MiaPaCa2 cells treated with $100 \mu \mathrm{M}$ of the GLUD1 inhibitor, EGCG, for $48 \mathrm{~h}$ followed by 2 -h B-lap dose response. Relative cell viability represents mean of CellTiter-Glo survival assay $48 \mathrm{~h}$ after $ß$-lap treatment plotted as percentage treated/control (T/C) \pm SE from sextuplicate samples. c Clonogenic survival assay of normal lung fibroblast cell line, IMR90, pre-treated with $\pm 500 \mathrm{nM}$ BPTES for $48 \mathrm{~h}$ followed by $2 \mathrm{~h}$ of $\mathrm{B}$-lap treatment. Data represent survival means \pm SE from quadruplicate samples. d MiaPaCa2, ASPC1, and HPAFII PDA cell lines were pre-treated with $\pm 500 \mathrm{nM}$ BPTES for $48 \mathrm{~h}$, either $3 \mathrm{mM}$ oxaloacetate (OAA) or $3 \mathrm{mM}$ dimethyl malate for the last $24 \mathrm{~h}$, and $2.5 \mu \mathrm{M}$ B-lap for $2 \mathrm{~h}$. Relative cell viability represents means of CellTiter-Glo survival assay $48 \mathrm{~h}$ after treatment plotted as percentage $(T / C) \pm S E$ from sextuplicate samples. e Various cancer cell lines pre-treated with $\pm 500 \mathrm{nM}$ BPTES (sub-growth inhibitory) for $48 \mathrm{~h}$ followed by the addition of $2.5 \mu \mathrm{M}$ B-lap for $2 \mathrm{~h}$. Mutant (mt) KRAS lines: A549 non-small cell lung (NSCL), PL45 PDA, NQO1 expressing S2-013 (S2+) PDA, NQO1 expressing MDA-MB-231 (231+) triple-negative breast, H2122 NSCL, NQO1-deficient S2-013 (S2-) PDA, and NQO1 deficient MDA-MB-231 (231-) triple-negative breast cancer cells. Wild-type (wt) KRAS lines: Hs766T PDA, BxPC3 PDA, MCF7 breast, as well as NQO1+ H596 NSCL and H661 NSCL cancer cell lines 
GLS1 inhibition attenuates anti-oxidant defenses and increases susceptibility to ß-lap-induced DNA damage We observed a dose-dependent increase in NADP + /NADPH ratios, a proxy for the cell's oxidative state, in MiaPaCa2 cells exposed to $ß$-lap alone, reaching fourfold higher levels versus baseline found in untreated cells (Fig. 4a). With BPTES pre-treatment, we noted a sevenfold increase in NADP+/NADPH ratios in ß-lap-exposed cells versus baseline levels in untreated $\mathrm{MiaPaCa} 2$ cells (Fig. $4 \mathrm{a}$ ) and an $\sim$ twofold higher level than in cells exposed to ß-lap alone. BPTES pre-treatment (500 nM, $48 \mathrm{~h}$ ) also significantly lowered reduced glutathione (GSH) levels in Mia$\mathrm{PaCa} 2$ cells compared to DMSO vehicle alone (>twofold; Additional file 3: Figure S3A), and extracellular $\mathrm{H}_{2} \mathrm{O}_{2}$ production was dramatically increased after BPTES plus ß-lap treatment in a time- and dose-dependent manner (Fig. 4b, Additional file 3: Figure S3B). Additionally, total intracellular ROS levels were dramatically increased after BPTES plus ß-lap treatment (Additional file 3: Figure S3C). Consistent with the kinetics of $\mathrm{H}_{2} \mathrm{O}_{2}$ production, we observed a decrease in the minimum time to death [31, 32] for ß-lapinduced lethality in MiaPaCa2 with BPTES pre-treatment (Fig. 4c). This effect on clonogenic survival could be rescued with the anti-oxidant reduced diethyl-ester GSH in ß-lap-exposed MiaPaCa2, ASPC1, HPAFII, and MPanc96 cells after $48 \mathrm{~h}$ pre-treatment with BPTES plus 2 h with ß-lap (Fig. 4d).

Pre-treatment with BPTES followed by exposure to $ß$ lap synergistically increased total DNA lesions in ASPC1 cells, as assessed by alkaline comet assay immediately after

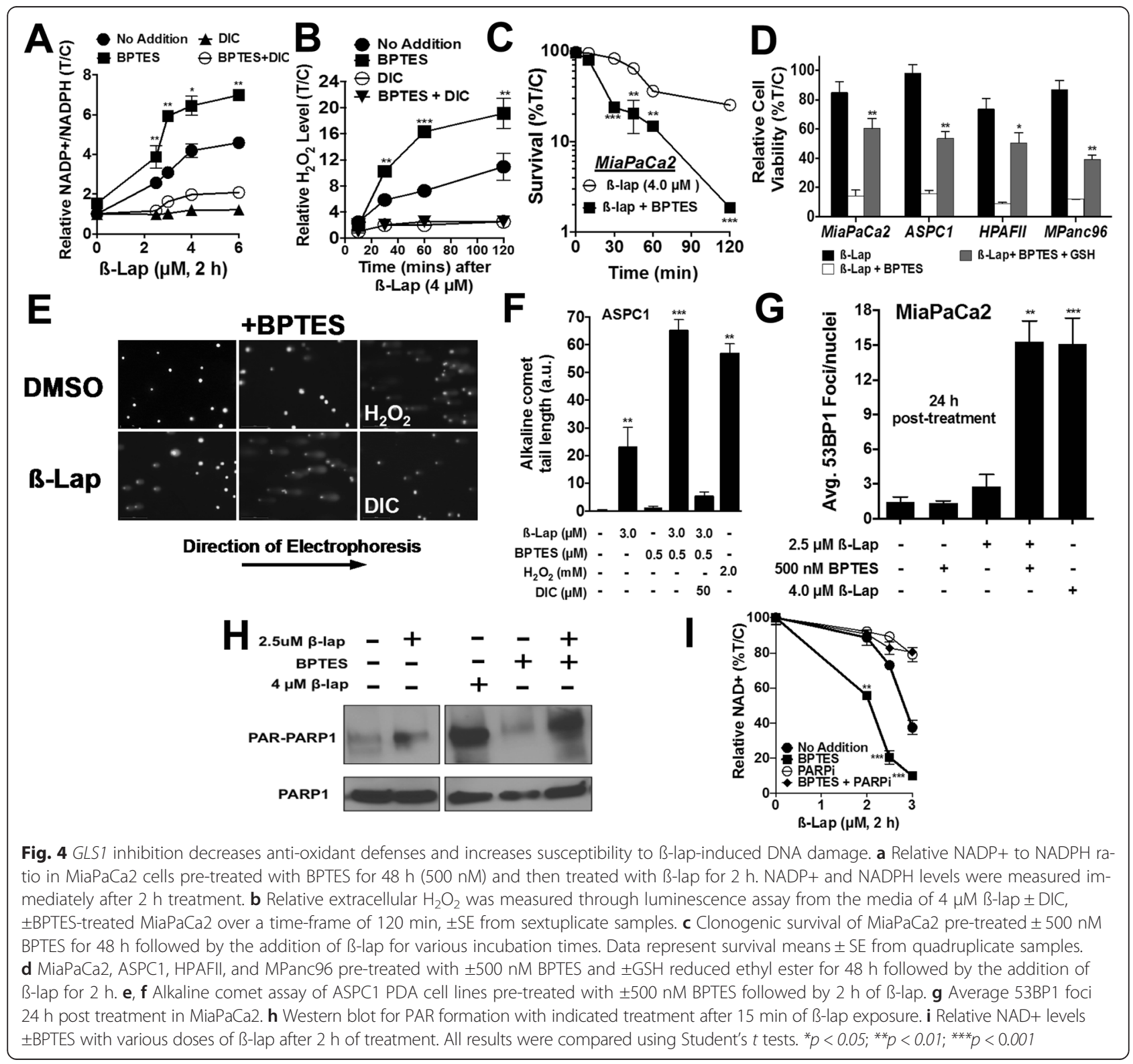


2-h treatment, and DNA double-strand break (DSB) formation in MiaPaCa2 cells, monitored by 53BP1 foci formation $24 \mathrm{~h}$ after treatment (Figs. 4e-g). Furthermore, pre-treatment with BPTES followed by treatment with $B$ lap dramatically increased PARP hyperactivation noted by concomitant NAD+ depletion and PAR formation, which was blocked with the addition of the PARP inhibitor, Rucaparib (AG014699), in MiaPaCa2 cells compared to either treatment alone (Fig. $4 \mathrm{~h}, \mathrm{i}$ ). These data indicate that PDA cells are reliant on glutamine for redox balance and that the disrupted redox state enhances ß-lap-induced DNA damage and PARP-driven metabolic catastrophe.

\section{GLS1 inhibition sensitizes PDA to B-lap in vivo}

To determine whether pharmacologic inhibition of GLS1 in combination with $B$-lap would lead to synergistic inhibition of PDA tumor growth in vivo, we utilized the clinical formulation of B-lap (ARQ761), hydroxypropyl beta cyclodextrin travel (HPßCD)- $B$-lap, and the orally available GLS1 inhibitor, CB-839, provided by Calithera Biosciences. Both compounds are in separate phase I/II clinical trials for a variety of cancer types (NCT01502800,
NCT02071862, NCT02071888, and NCT02071927) [11]. CB-839 was employed for these studies because BPTES has poor metabolic stability and low solubility in vivo [11]. First, we confirmed that CB-839 pre-treatment, like BPTES, also sensitized PDA cell lines in vitro to ß-lap in the MiaPaCa2 and ASPC1 lines (Fig. 5a). Next, we generated subcutaneous tumors from human $\mathrm{MiaPaCa} 2$ cells injected into the right hind limb in $\mathrm{Nu} / \mathrm{Nu}$ female athymic mice and allowed the tumors to grow to a volume of $100 \mathrm{~mm}^{3}$ before beginning treatment. The mice were sacrificed when tumor volumes reached $1000 \mathrm{~mm}^{3}$, as per the Institutional Animal Care and Use Committee (IACUC) regulations.

The animals received either vehicle (i.e., $\mathrm{HP} ß C D$ ) or CB-839 $(200 \mathrm{mg} / \mathrm{kg})$ by oral gavage twice a day for 10 days, with or without a sub-efficacious dose of $ß$-lap $(25 \mathrm{mg} / \mathrm{kg}$ ) administrated intravenously (IV) every other day (Fig. 5b, arrows) [33]. After only one regimen of treatment (10 days), we found that the mice treated with CB-839 plus ß-lap displayed significantly delayed tumor growth compared to either agent alone through day 60 . Importantly, we noted that neither CB-839 $(200 \mathrm{mg} / \mathrm{kg})$
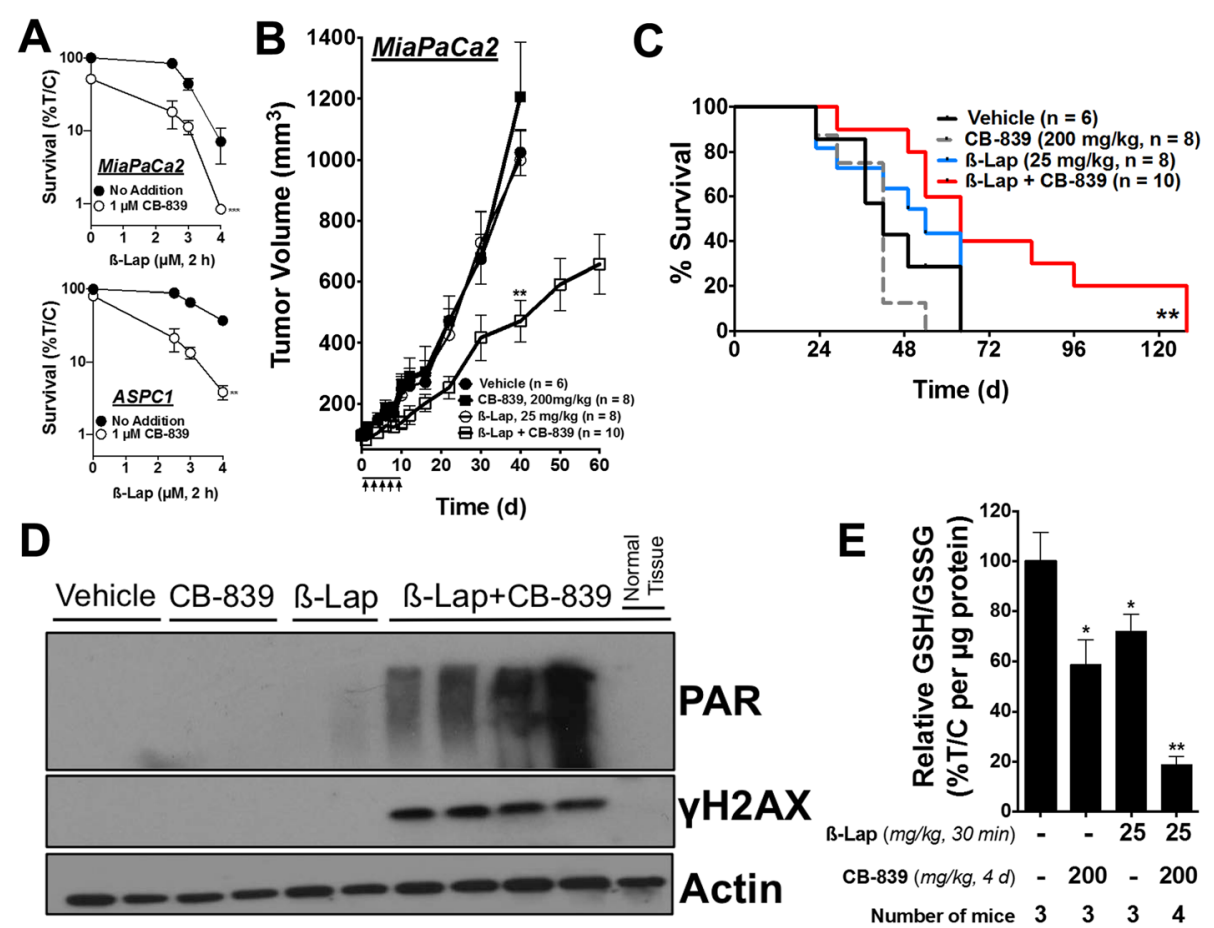

Fig. 5 GLS1 inhibition sensitizes pancreatic cancer to ß-lap in vivo. a Clonogenic survival of MiaPaCa2 and ASPC1 cells pre-treated with $1 \mu$ M CB-839 for $48 \mathrm{~h}$ followed by $2 \mathrm{~h}$ of B-lap dose response. $\mathbf{b}$ Subcutaneous tumors grown from MiaPaCa2 cells in nude mice were allowed to reach a volume of $100 \mathrm{~mm}^{3}$, after which, the mice were treated every other day with vehicle (HPBCD, $n=6$ ), sub-efficacious dose of CB-839 (oral gavage, $200 \mathrm{mg} / \mathrm{kg}$, twice daily for 10 days, $n=8$ ), sub-efficacious dose of B-lap (IV, $25 \mathrm{mg} / \mathrm{kg}, n=8$ ) or sub-efficacious doses of CB-839 and B-lap ( $n=10$ ) for a total of five doses (arrows). Tumor growth was monitored until tumors reached $1000 \mathrm{~mm}^{3}$. Error bars, SEM. c Survival of tumor-bearing mice represented as a Kaplan-Meier plot. The mice were sacrificed when tumors reached $1000 \mathrm{~mm}^{3}$. Statistically analyzed with the log-rank test for trend. $\mathbf{d}$ Western blot of PAR and $\mathrm{YH} 2 \mathrm{AX}$ from a set of tumors harvested $30 \mathrm{~min}$ after treatment with 3 -lap \pm CB-839 (4-day treatment), $n=3$ tumors per group, same doses as above. e Relative GSH/GSSG ratio in treated groups normalized to microgram of tumor protein. Unless stated otherwise, all results were compared using Student's $t$ tests. ${ }^{*} p<0.05$; ${ }^{* *} p<0.01$; ${ }^{* * *} p<0.001$ 
nor ß-lap $(25 \mathrm{mg} / \mathrm{kg}$ ) administered alone significantly altered tumor growth (Fig. 5b). Individual tumors are presented in waterfall plots in Additional file 4: Figure S4A. Notably, combination treatment did not decrease mouse weights when compared to vehicle-treated mice (Additional file 4: Figure S4B). Long- or short-term toxicities, including hemolysis and methemoglobinemia, were not observed.

The mice were sacrificed when their original body weights dropped by one third, tumor volumes exceeded $1000 \mathrm{~mm}^{3}$, or when tumors began to ulcerate or impede normal motion. Kaplan-Meier curves showed a significant anti-tumor effect of the drug combination (Fig. 5c). The treatment with a sub-efficacious dose of ß-lap $(25 \mathrm{mg} / \mathrm{kg})$ resulted in a median survival of 49 days, while the vehicle-treated group displayed a median survival time of 39.5 days (Fig. 5c). The treatment with a sub-efficacious dose of CB-839 resulted in a median survival time of 42 days. The treatment with the 3 -lap plus CB-839 combination resulted in a median survival of 64 days, significantly extending median survival by 24.5 days compared to the vehicle (HPßCD)-treated group (Fig. 5c).

Importantly, to ensure that the anti-tumor efficacy we observed was due to on-target effects of both drugs and by the same mechanism of action observed in vitro, we analyzed the pharmacodynamics profile of each agent alone and in combination. Briefly, the MiaPaCa2 tumorbearing mice received either vehicle (HPßCD) alone or $200 \mathrm{mg} / \mathrm{kg}$ CB-839 by oral gavage twice a day for 4 days, with or without a single sub-efficacious dose of 3 -lap at $25 \mathrm{mg} / \mathrm{kg}$ IV on day 4. After the last dose of CB-839 and 30 min after $ß$-lap injection, the mice were sacrificed and the tumor tissue was harvested. Tumor glutamate levels were measured from multiple animals for each treatment condition (Additional file 4: Figure S4C). CB-839 and CB839 plus B-lap-treated mice displayed significantly lower overall tumor glutamate levels when compared to vehicle or ß-lap alone treatments, consistent with GLS1 inhibition in vivo (Additional file 4: Figure S4C).

We then immunoblotted tumor tissue lysates for PAR polymer formation as a proxy for PARP hyperactivity and $\gamma \mathrm{H} 2 \mathrm{AX}$ to assess DNA DSBs (Fig. 5d, see quantification in Additional file 4: Figure S4D) [33]. Consistent with our results in vitro, we found that the mice treated with the combination of 3 -lap plus CB-839 displayed dramatically increased PAR and $\gamma \mathrm{H} 2 \mathrm{AX}$ formation relative to all other groups (Fig. 5d). Additionally, we harvested liver tissue ("normal tissue" in Fig. 5d) from the mice after exposure to the combination-treated mice and found no evidence of PAR or $\gamma \mathrm{H} 2 \mathrm{AX}$ formation (Fig. 5d), consistent with a lack of response to $ß$-lap in normal tissues $[24,33]$. Notably, the liver contains the highest levels of NQO1 in the mouse (not in human livers) and is used as a surrogate for normal tissue responses to NQO1-bioactivatable drugs.
Next, to determine the redox status of tumors after treatment, we measured the GSH to glutathione disulfide (GSSG) ratio of tumor lysates after vehicle alone or CB-839, with or without 30-min B-lap treatment. Interestingly, we found that the GSH to GSSG ratios were significantly decreased in the single-arm CB-839 or ß-lap-treated mice (Fig. 5e). Moreover, the combination treatment resulted in an even greater decrease in GSH, monitored by the GSH to GSSG ratio (Fig. 5e). Taking our pharmacodynamics observations and antitumor studies together, these data demonstrate that modulating glutamine metabolism in PDA in vivo results in a significantly decreased anti-oxidant defense state. As a result, the drug combination significantly sensitized NQO1-expressing tumors, but not associated normal tissue, to ROS induction from $ß$-lap leading to DNA damage, PARP hyperactivation, and tumor-selective death.

\section{Discussion}

Tumor cells display unique metabolic alterations that impact the biological behavior of the tumor and have therapeutic implications [34, 35]. A well-documented example of such an alteration is the increased utilization of glutamine in a variety of human tumors, including lung, prostate, lymphoma, and PDA $[8,15,36,37]$. Glutamine has pleiotropic roles in the cell that include the regulation of autophagy, signal transduction, anabolic growth, and redox balance [9]. In PDA, mutant KRAS promotes the reprogramming of glutamine metabolism through a GOT1- and GOT2-mediated transamination pathway instead of the pathway catalyzed by GLUD1 [8]. This transamination pathway is necessary to maintain redox balance in PDA tumors through maintenance of reduced NADPH and GSH levels (Fig. 1a) [2, 8, 9]. While this understanding provides insight into PDA metabolism, how to most appropriately exploit this metabolic vulnerability had remained to be determined. Previous work revealed that targeting this pathway alone resulted in cytostasis, driving compensatory metabolic resistance pathways in the tumor. In addition, potential systemic toxicities remain to be determined.

Genes involved in the glutamine-dependent transamination pathway (GLS1, GOT1/2, ME1) and NQO1, but not GLUD1, are highly expressed in PDA relative to other cancers (Fig. 1b). Clinical PDA cases expressing a high GOT1 to GLUD1 or GOT2 to GLUD1 ratio in the tumor have worse outcomes (Fig. 1c, Additional file 1: Figure S1B). Here, we found that genetic or pharmacological inhibition of this pathway sensitizes PDA to the NQO1-bioactivatable drug, $ß$-lap. Addition of $ß$-lap in combination with GLS1 inhibition resulted in enhanced tumor-cell specificity and efficacy in PDA cell lines, not typically found with GLS1 inhibition alone. Consistent with prior reports of differential usage of 
glutamine in mutant KRAS versus wild-type KRAS cancers $[4,8,30,38]$, we found that mutant $K R A S$ cell lines, but not wild-type KRAS PDA, lung, and breast cancer, were sensitized to $ß$-lap upon pre-treatment with BPTES. These data suggest that mutant, compared to wild-type, KRAS cancers may also rely on glutamine to maintain redox balance, though this may not necessarily involve the transamination pathway discussed. Future studies to elucidate this mechanism will be needed.

When considering the ROS burst generated from NQO1-bioactivatable drugs, as evidenced by 3 -lap's $\mathrm{H}_{2} \mathrm{O}_{2}$ production profile, cells require an equally robust antioxidant response to suppress ROS accumulation. Thus, one can imagine that a competition between the rates of engagement of the anti-oxidant machinery and the production of ROS determines the fate of cancer cells within a tumor following NQO1-bioactivatable drug treatment. Notably, we previously reported that catalase (an essential enzyme for the detoxification of $2 \mathrm{H}_{2} \mathrm{O}_{2}$ to $2 \mathrm{H}_{2} \mathrm{O}+\mathrm{O}_{2}$ ) expression was significantly downregulated in tumor tissue relative to normal tissue, and that the NQO1 to catalase ratio is markedly increased in tumor tissue. Moreover, addition of PEGylated catalase significantly protected breast cancer cell lines from ß-lap-induced lethality [25]. To maintain redox balance, PDA may utilize glutamine metabolism to compensate for this decrease in catalase. Indeed, addition of BPTES to NQO1-overexpressing cancer cells decreased NADPH and GSH anti-oxidant defenses, which would otherwise counteract the $ß$-lapinduced ROS burst and thus lead to enhanced DNA damage, PARP-driven NAD+ and ATP depletion, and metabolic catastrophe. Mechanistically, this occurred in a near-identical manner to the caspase-independent, $\mu$ calpain-mediated cell death pathway induced by lethal doses of $ß$-lap alone [39], only at lower doses, which act to augment the therapeutic window for $B$-lap.

For our in vivo studies, we utilized the GLS1 inhibitor CB-839, as this compound has far greater solubility and stability in animals than BPTES [11]. A single regimen of CB-839 plus $ß$-lap in the tumor-bearing mice slowed tumor progression and significantly improved survival compared to the animals treated with either agent alone. Pharmacodynamics analysis revealed that the mechanism of synergy with combination treatment in vivo was consistent with the in vitro results. In sum, these results are promising but preliminary in nature, given their reliance on subcutaneous PDA cell line-derived xenografts, as these models may overestimate the efficacy of therapeutic regimens [40]. Future work will test combination treatment regimens in genetically engineered PDA mouse models [41]. We should note, however, that subcutaneous xenograft models do represent a challenging microenvironment for pancreatic cancer studies due to the hypoxic environment and limited blood supply.

\section{Conclusions}

Novel therapies are desperately needed for patients with PDA. By combining GLS1 inhibition (e.g., CB-839) and NQO1-bioactivatable drugs (e.g., $\beta$-lap, ARQ761), we exploit the reliance of PDA on glutamine for redox balance, as well as the tumor-selective overexpression of NQO1 through the use of a unique agent that is bioactivated to induce cell death. Combination treatment with GLS1 inhibitors plus ß-lap addresses limitations associated with either agent alone. Namely, this combination is expected to enhance efficacy at well-tolerated doses of these drugs; the concentrations used in this study are relevant to those that are achievable in patients (NCT01502800, NCT02071862, NCT02071888, and NCT02071927) [11, 42]. Furthermore, we noted that CB-839 plus B-lap combination-treated animals did not display increased cytotoxicity in normal liver tissue, based on a lack of PAR and DNA damage formation. These results reinforce the necessity for NQO1 expression to achieve cell death. Collectively, these findings illustrate a rational combination strategy to target PDA dependence on glutamine, by GLS1 inhibition, in combination with an NQO1-bioactivatable drug and provide the proof-of-concept validation to warrant analysis in a larger pre-clinical context.

\section{Methods}

\section{Cell culture}

A549, MiaPaCa2, DAN-G, SW1990, MPanc96, SUIT2013-NQO1+/-, HPAFII, ASPC1, PL45, H596, Hs766T, BxPC3, MBA-MD-231+/-, and MCF7 cell lines were obtained from ATCC, tested for mycoplasma contamination and grown in complete Dulbecco's Modified Eagle's Medium (DMEM) with $10 \%$ fetal bovine serum (FBS). H2122 and H661 cell lines were a generous gift from Dr. John Minna at UTSW. SUIT2-013 and MDAMB-231 NQO1 deficient and proficient cell lines were generated as previously described [24]. DMEM (glutamine free) was purchased from Sigma-Aldrich. FBS was purchased from Fischer Scientific. Glutamine, GSH-reduced ethyl ester, OAA, and dimethyl malate were purchased from Sigma. IMR90 cells lines were grown in MEM with $10 \%$ FBS. All cells were incubated at $37^{\circ}$ with $5 \% \mathrm{CO}_{2}$.

\section{Reagents and chemicals}

ß-Lap was synthesized, purified, and prepared at $50 \mathrm{mM}$ stock solution as previously described [18]. BPTES and DIC were purchased from Sigma-Aldrich (St Louis, MO). CB-839 was kindly provided by Calithera Biosciences (San Francisco, CA).

\section{Survival assays}

For clonogenic survival assays, the cells were plated in 10 -cm plates at $2 \times 10^{5}$ cells per plate and pre-treated 
with \pm BPTES for $48 \mathrm{~h}$, then treated with the appropriate ß-lap doses for $2 \mathrm{~h}$. The cells were then trypsinized and plated onto six-well plates at 100, 500, or 1000 cells per well in $2 \mathrm{~mL}$ of complete media. After 7 days of proliferation, the plates were washed in PBS and colonies were fixed in methanol/crystal violet; all clonogenic assay results are represented as \% Survival (treated/control, T/C). Cell viability was also assessed using the CellTiter-Glo assay (Promega). The cells were plated and pre-treated with \pm BPTES as described above. The cells were then plated at $6 \times 10^{4}$ cells per well in a 96-well plate in $100 \mu \mathrm{L}$ of media. The following day, the cells were treated with the appropriate doses of $\mathrm{B}$-lap for $2 \mathrm{~h}$, and then ATP levels were checked $48 \mathrm{~h}$ after treatment; cell viability is represented as Relative Cell Viability \% (T/C). Relative cell survival was also assessed using 7-day DNA assays as previously described and reported as Relative Survival \% (T/C) [43]. Results are reported as means \pm standard error (SE) from at least three independent experiments done in sextuplicate. This dose of $\beta$-lap $(4 \mu \mathrm{M}, 2 \mathrm{~h})$ typically yields $80-90 \%$ lethality in $\mathrm{MiaPaCa} 2$ cell lines across these assays.

\section{Glutathione, $\mathrm{NAD}(\mathrm{P}) \mathrm{H}, \mathrm{H}_{2} \mathrm{O}_{2}$, and total ROS assays}

The following assays were purchased from Promega: GSH/GSSG-Glo, NAD/NADH-Glo, NADP/NADPH-Glo, and ROS-Glo and were used as instructed by the manufacturer. The total intracellular ROS levels were detected using the cell-permeable fluorogenic probe CellROX (Molecular Probes) that emits red fluorescence upon oxidation by ROS. Briefly, the cells were incubated with $5 \mu \mathrm{M}$ CellROX ${ }^{\circ}$ and $ß$-lap for $30 \mathrm{~min}$ at $37^{\circ} \mathrm{C}$ followed by fixation in $4 \%$ paraformaldehyde. ROS generation was analyzed by flow cytometry.

\section{siRNA knockdown}

siRNA was transiently transfected into the cells using Opti-MEM and Lipofectamine RNAiMAX for $48 \mathrm{~h}$ (Life Technologies) for the following enzymes: GLS1, ONTARGET plus GLS1 SMARTpool; GLS1, 5'-GAUG GAUUGUUGUAAUGGU-3'; GLUD1, 5'-CUCACUAU CCUCUUCACAU-3'; GOT1, 5'CUCUAACCCUGAG CUCUUU-3'; ME1, 5' -GACACUUAGAUUAAGAUUU3'; and GOT2, 5'GCCUUUAAGAGGGACACCA-3'. All siRNAs were purchased from Sigma. After $48 \mathrm{~h}$ of incubation, the cells were detached with trypsin/EDTA (Life Technologies) and seeded for treatment assays or lysed for analysis of knockdown efficiency. Growing cells in OptiMEM and Lipofectamine for $48 \mathrm{~h}$ increased their sensitivity to ß-lap in the siScr group alone.

\section{Western blot}

Western blots were carried out as previously described [25]. Primary antibodies for protein detection included:
GLS1 (ab93434, Abcam), PARP1 (F-2, Santa Cruz), PAR (Trevigen, Gaithersburg, MD), Actin (C-2, Santa Cruz), small subunit calpain (EPR3324, Abcam), and 53BP1 (Bethyl Laboratories).

\section{Human xenograft anti-tumor, pharmacodynamics assays}

The athymic $\mathrm{Nu} / \mathrm{Nu}$ nude female mice $(18-20 \mathrm{~g})$ were commercially obtained (Harlan). Human xenografts were generated by injecting $2 \times 10^{6} \mathrm{MiaPaCa} 2$ cells subcutaneously in PBS/Matrigel into 6-week-old mice. The tumors were measured at indicated times with digital calipers (Fisher Scientific), and tumor volumes calculated (length $\times$ width $^{2} \times 0.5$ ). The treatments were initiated when subcutaneous tumors reached an average size of $\geq 100 \mathrm{~mm}^{3}$. The mice were treated with CB- 839 by oral gavage, ß-lap (as HPßCD-ß-lap) by IV (retro-orbital) or both or with vehicle (HPßCD; 1:9, v/v; SigmaAldrich) as a control. The treatment regimens consisted of a total of five doses of $ß$-lap, with one dose being administered every other day for 10 days. CB-839 was administered twice a day, everyday for 10 days. The mice bearing subcutaneous tumors were treated with nontoxic doses $(200 \mathrm{mg} / \mathrm{kg})$ of CB-839, with or without a sub-efficacious dose of HPßCD- $\beta$-lap $(25 \mathrm{mg} / \mathrm{kg})$. The mice bearing subcutaneous tumors were sacrificed when tumors reached $>1000 \mathrm{~mm}^{3}$ as per IACUC. Overall survival was assessed through Kaplan-Meier curves using the log-rank test for trend for statistical significance. The mice were weighed three times per week during and after the drug treatment period with no toxicities observed. All animal studies were performed in accordance under protocols approved by the Institutional Animal Care and Use Committee of UT Southwestern Medical Center.

\section{Statistics}

All graphs are plotted as mean with error bars denoting standard deviation. Regression analysis, ANOVA, and twotailed Student $t$ tests for statistical significance with Holm/ Sidak multiple comparison correction were performed in GraphPad Prism 6. $p$ values were expressed throughout by ${ }^{*} p<0.05,{ }^{* * *} p<0.01,{ }^{* * * *} p<0.001$, and ${ }^{* * * * *} p<0.0001$.

\section{Additional files}

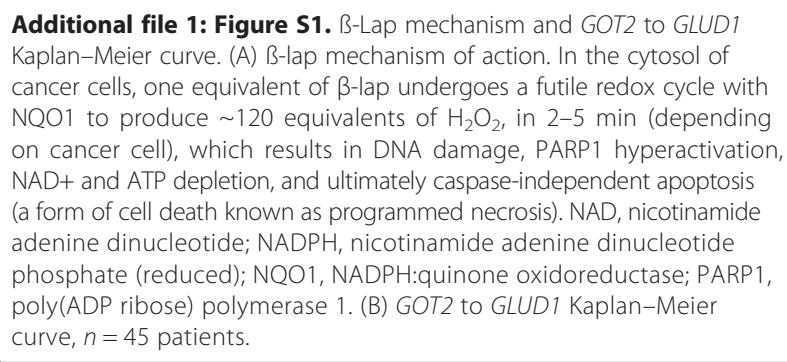


Additional file 2: Figure S2. BPTES sensitivity. (A) Growth inhibition in MiaPaCa2 cells, as monitored by loss of ATP, to short-term (48 h) or long-term (96 h) BPTES treatments.

Additional file 3: Figure S3. Combination treatment leads to increased $\mathrm{ROS}$ by depleting reduced glutathione. (A) Relative GSH levels $\pm 500 \mathrm{nM}$ BPTES pre-treatment followed by B-lap treatment. (B) Relative $\mathrm{H}_{2} \mathrm{O}_{2}$ production with $\pm 500 \mathrm{nM}$ BPTES pre-treatment followed by B-lap treatment. (C) CellROX ${ }^{\odot}$ quantification of total ROS at 15 min of $\pm 4 \mu \mathrm{M}$ B-lap, \pm DIC, and \pm BPTES (48-h pre-treatment).

Additional file 4: Figure S4. Assessment of drug effects on PDA tumors and animals. (A) Change in tumor volumes per animal at day 22 compared to day 1. (B) Mouse weights during treatment for vehicle $(n=6)$, CB-839 ( $n=8)$, B-lap $(n=8)$ and B-lap plus CB-839 $(n=10)$. (C) Tumor glutamate levels, $n=3$ mice per group. (D) Quantification of relative PAR and $\gamma \mathrm{H} 2 \mathrm{AX}$ from tumor tissue lysate according to the western blot in Fig. 5 d.

\section{Abbreviations}

ADP: adenosine diphosphate; ATP: adenosine triphosphate; DIC: dicoumarol, GEMM: genetically modified mouse model; GLS1: mitochondrial glutaminase 1; GLUD1: glutamate dehydrogenase 1; GOT1: cytoplasmic glutamate oxaloacetate transaminase 1; GOT2: mitochondrial glutamate oxaloacetate transaminase 2; GSH: glutathione (reduced); KRAS: Kirsten rat sarcoma viral oncogene homolog; MDH1: cytosolic malate dehydrogenase 1; ME1: malic enzyme 1; NAD: nicotinamide adenine dinucleotide; NADPH: nicotinamide adenine dinucleotide phosphate (reduced); NQO1: NADPH:quinone oxidoreductase; PAR: poly(ADP ribose); PARP: poly(ADP ribose) polymerase; PD: pharmacodynamics; PDA: pancreatic ductal adenocarcinoma; PK: pharmacokinetics; ROS: reactive oxygen species.; B-lap: B-lapachone.

\section{Competing interests}

ACK is a Consultant for Forma Therapeutics. LCC owns equity in and receives compensation from Agios Pharmaceuticals. LCC serves on the Board of Directors and Scientific Advisory Board of Agios Pharmaceuticals. RJD is also on the Scientific Advisory Board of Agios Pharmaceuticals.

\section{Authors' contributions}

GC designed and carried out the in vitro and in vivo experiments and drafted the manuscript. ZM aided in the design of in vitro experiments, reproduced the NAD+/ATP measurement studies, and aided in drafting the manuscript. $X \mathrm{~L}$ conducted the initial metabolic studies. $\mathrm{ML}$ aided in the in vivo studies. AA helped interpret the metabolic studies. MP reproduced the glutamine deprivation survival studies. YZ conducted the survival and gene expression analysis. YX aided in the survival and gene expression analysis. SB aided in the drafting of the manuscript and in the interpretation of in vitro and in vivo findings. PPS aided in the editing and drafting of the manuscript. ACK helped design the in vivo studies and aided in drafting the manuscript. RJD helped design the metabolic studies, interpret the results, and draft the manuscript. LCC helped design the studies and edit the manuscript. CAL interpreted the data, designed the studies, provided essential reagents, and helped edit and draft the manuscript. DAB designed and drafted the manuscript and supervised all studies. All authors read and approved the final manuscript.

\section{Authors' information}

Not applicable.

\section{Acknowledgements}

Support for use of Shared Resources within the Simmons Comprehensive Cancer Center was funded by NIH/NCI grant\# 5P30CA142543. Support for these studies was provided by grant $\mathrm{NIH} / \mathrm{NCl}$ R01 CA102792 to DAB and grants $\mathrm{NIH}$ R01 CA157996 05 and RP130272 (CPRIT) to RJD. This work was also supported by a PanCan Translational Award\# 15-65-25-BOOT and AACR/PanCan/Rising Tide/Gateway Clinical Continuation Grant \#14-65-25-BOOT to DAB. CAL is supported

by a Pancreatic Cancer Action Network Pathway to Leadership Award. Finally, this paper is dedicated to the memories of Mrs. Sarah Hildebrand and Mrs. Rosemarie Bouley for their strong support of our research.

\section{Author details}

${ }^{1}$ Department of Pharmacology, University of Texas Southwestern Medical Center, 6001 Forest Park Drive, Dallas 75390-8807TX, USA. ²Radiation Oncology, University of Texas Southwestern Medical Center, Dallas, TX, USA. ${ }^{3}$ Touchstone Diabetes Center, Simmons Comprehensive Cancer Center, University of Texas Southwestern Medical Center, Dallas, TX, USA. ${ }^{4}$ Department of Internal Medicine, Weill Cornell Medical College, 413 East 69th Street, BB-1362, New York, NY 10021, USA. ${ }^{5}$ Department of Bioinformatics and Biostatistics, Clinical Sciences, UT Southwestern Medical Center, 5323 Harry Hines Boulevard, Dallas, TX 75390, USA. ${ }^{6}$ Department of Medicine, Weill Cornell Medical College, 413 East 69th Street, BB-1362, New York, NY 10021, USA. ${ }^{7}$ Children's Medical Center Research Institute, UT Southwestern Medical Center, 5323 Harry Hines Blvd, Dallas, TX 75390, USA. ${ }^{8}$ Department of Radiation Oncology, Division of Genomic Stability and DNA Repair, Dana-Farber Cancer Institute, Boston, MA 02215, USA. ${ }^{9}$ Department of Molecular and Integrative Physiology, University of Michigan, Ann Arbor, MI 48109, USA. ${ }^{10}$ Internal Medicine, Division of Gastroenterology, University of Michigan, Ann Arbor, Ml 48109, USA.

Received: 27 June 2015 Accepted: 17 September 2015

Published online: 12 October 2015

\section{References}

1. Hidalgo M. Pancreatic cancer. N Engl J Med. 2010;362(17):1605-17.

2. Bryant KL, Mancias JD, Kimmelman AC, Der CJ. KRAS: feeding pancreatic cancer proliferation. Trends Biochem Sci. 2014;39(2):91-100.

3. Cox AD, Fesik SW, Kimmelman AC, Luo J, Der CJ. Drugging the undruggable RAS: mission possible? Nat Rev Drug Discov. 2014;13(11):828-51.

4. Kong B, Qia C, Erkan M, Kleeff J, Michalski CW. Overview on how oncogenic Kras promotes pancreatic carcinogenesis by inducing low intracellular ROS levels. Front Physiol. 2013;4:246.

5. Lyssiotis CA, Son J, Cantley LC, Kimmelman AC. Pancreatic cancers rely on a novel glutamine metabolism pathway to maintain redox balance. Cell Cycle. 2013;12(13):1987-8.

6. Kamphorst JJ, Nofal M, Commisso C, Hackett SR, Lu W, Grabocka E, et al. Human pancreatic cancer tumors are nutrient poor and tumor cells actively scavenge extracellular protein. Cancer Res. 2015;75(3):544-53.

7. Ying H, Kimmelman AC, Lyssiotis CA, Hua S, Chu GC, Fletcher-Sananikone E, et al. Oncogenic Kras maintains pancreatic tumors through regulation of anabolic glucose metabolism. Cell. 2012;149(3):656-70.

8. Son J, Lyssiotis CA, Ying H, Wang X, Hua S, Ligorio M, et al. Glutamine supports pancreatic cancer growth through a KRAS-regulated metabolic pathway. Nature. 2013;496(7443):101-5.

9. Shanware NP, Mullen AR, DeBerardinis RJ, Abraham RT. Glutamine: pleiotropic roles in tumor growth and stress resistance. J Mol Med. 2011;89(3):229-36.

10. Shukla K, Ferraris DV, Thomas AG, Stathis M, Duvall B, Delahanty G, et al. Design, synthesis, and pharmacological evaluation of bis-2-(5-phenylacetamido1,2,4-thiadiazol-2-yl)ethyl sulfide 3 (BPTES) analogs as glutaminase inhibitors. J Med Chem. 2012;55(23):10551-63.

11. Gross MI, Demo SD, Dennison JB, Chen L, Chernov-Rogan T, Goyal B, et al. Antitumor activity of the glutaminase inhibitor CB-839 in triple-negative breast cancer. Mol Cancer Ther. 2014;13(4):890-901.

12. Stalnecker CA, Ulrich SM, Li Y, Ramachandran S, McBrayer MK, DeBerardinis RJ, et al. Mechanism by which a recently discovered allosteric inhibitor blocks glutamine metabolism in transformed cells. Proc Natl Acad Sci U S A. 2015;112(2):394-9.

13. Emadi A, Jun SA, Tsukamoto T, Fathi AT, Minden MD, Dang CV. Inhibition of glutaminase selectively suppresses the growth of primary acute myeloid leukemia cells with IDH mutations. Exp Hematol. 2014;42(4):247-51.

14. Zhdanov AV, Waters AH, Golubeva AV, Dmitriev RI, Papkovsky DB. Availability of the key metabolic substrates dictates the respiratory response of cancer cells to the mitochondrial uncoupling. Biochim Biophys Acta. 2014;1837(1):51-62.

15. Le A, Lane AN, Hamaker M, Bose S, Gouw A, Barbi J, et al. Glucoseindependent glutamine metabolism via TCA cycling for proliferation and survival in B cells. Cell Metab. 2012;15(1):110-21.

16. Seltzer MJ, Bennett BD, Joshi AD, Gao P, Thomas AG, Ferraris DV, et al. Inhibition of glutaminase preferentially slows growth of glioma cells with mutant IDH1. Cancer Res. 2010;70(22):8981-7. 
17. Cheng T, Sudderth J, Yang C, Mullen AR, Jin ES, Mates JM, et al. Pyruvate carboxylase is required for glutamine-independent growth of tumor cells. Proc Natl Acad Sci U S A. 2011;108(21):8674-9.

18. Bey EA, Bentle MS, Reinicke KE, Dong Y, Yang CR, Girard L, et al. An NQO1- and PARP-1-mediated cell death pathway induced in non-small-cell lung cancer cells by beta-lapachone. Proc Natl Acad Sci U S A. 2007;104(28):11832-7.

19. Awadallah NS, Dehn D, Shah RJ, Russell Nash S, Chen YK, Ross D, et al. NQO1 expression in pancreatic cancer and its potential use as a biomarker. Appl Immunohistochem Mol Morphol. 2008;16(1):24-31.

20. Lyn-Cook BD, Yan-Sanders Y, Moore S, Taylor S, Word B, Hammons GJ. Increased levels of NAD(P)H: quinone oxidoreductase 1 (NQO1) in pancreatic tissues from smokers and pancreatic adenocarcinomas: A potential biomarker of early damage in the pancreas. Cell Biol Toxicol. 2006:22(2):73-80.

21. Lewis AM, Ough M, Hinkhouse MM, Tsao MS, Oberley LW, Cullen J. Targeting NAD(P)H:quinone oxidoreductase (NQO1) in pancreatic cancer. Mol Carcinog. 2005;43(4):215-24

22. Hartner LR MH LP, Mendelson D, Staddon AP, Chow W, Kovalyov O, Ruka W, et al. Phase 2 dose multi-center, open-label study of ARQ 501, a checkpoint activator, in adult patients with persistent, recurrent or metastatic leiomyosarcoma (LMS). J Clin Oncol. 2007;25:20521.

23. Zhang G, Schetter A, He P, Funamizu N, Gaedcke J, Ghadimi BM, et al. DPEP1 inhibits tumor cell invasiveness, enhances chemosensitivity and predicts clinical outcome in pancreatic ductal adenocarcinoma. PLoS One. 2012;7(2):e31507.

24. Cao L, Li LS, Spruell C, Xiao L, Chakrabarti G, Bey EA, et al. Tumor-selective, futile redox cycle-induced bystander effects elicited by NQO1 bioactivatable radiosensitizing drugs in triple-negative breast cancers. Antioxid Redox Signal. 2014;21(2):237-50.

25. Bey EA, Reinicke KE, Srougi MC, Varnes M, Anderson VE, Pink JJ, et al, Catalase abrogates beta-lapachone-induced PARP1 hyperactivation-directed programmed necrosis in NQO1-positive breast cancers. Mol Cancer Ther 2013;12(10):2110-20.

26. Bey EA, Reinicke KE, Srougi MC, Varnes M, Anderson V, Pink JJ, et al. Catalase abrogates beta-lapachone-induced PARP1 hyperactivation-directed programmed necrosis in NQO1-positive breast cancers. Molecular cancer therapeutics 2013, In Press.

27. Choo AY, Kim SG, Vander Heiden MG, Mahoney SJ, Vu H, Yoon SO, et al. Glucose addiction of TSC null cells is caused by failed mTORC1-dependent balancing of metabolic demand with supply. Mol Cell. 2010;38(4):487-99.

28. DeNicola GM, Karreth FA, Humpton TJ, Gopinathan A, Wei C, Frese K, et al. Oncogene-induced Nrf2 transcription promotes ROS detoxification and tumorigenesis. Nature. 2011;475(7354):106-9.

29. Gao P, Tchernyshyov I, Chang TC, Lee YS, Kita K, Ochi T, et al. c-Myc suppression of miR-23a/b enhances mitochondrial glutaminase expression and glutamine metabolism. Nature. 2009;458(7239):762-5.

30. Saqcena M, Mukhopadhyay S, Hosny C, Alhamed A, Chatterjee A, Foster DA. Blocking anaplerotic entry of glutamine into the TCA cycle sensitizes K-Ras mutant cancer cells to cytotoxic drugs. Oncogene. 2014;34(20):2672-80.

31. Bentle MS, Reinicke KE, Dong Y, Bey EA, Boothman DA. Nonhomologous end joining is essential for cellular resistance to the novel antitumor agent, beta-lapachone. Cancer Res. 2007;67(14):6936-45.

32. Bentle MS, Reinicke KE, Bey EA, Spitz DR, Boothman DA. Calcium-dependent modulation of poly(ADP-ribose) polymerase-1 alters cellular metabolism and DNA repair. J Biol Chem. 2006;281(44):33684-96.

33. Li LS, Bey EA, Dong Y, Meng J, Patra B, Yan J, et al. Modulating endogenous NQO1 levels identifies key regulatory mechanisms of action of betalapachone for pancreatic cancer therapy. Clin Cancer Res. 2011;17(2):275-85.

34. Hanahan D, Weinberg RA. Hallmarks of cancer: the next generation. Cell. 2011;144(5):646-74.

35. Cheong H, Lu C, Lindsten T, Thompson CB. Therapeutic targets in cancer cell metabolism and autophagy. Nat Biotechnol. 2012;30(7):671-8.

36. Brunelli L, Caiola E, Marabese M, Broggini M, Pastorelli R. Capturing the metabolomic diversity of KRAS mutants in non-small-cell lung cancer cells. Oncotarget. 2014;5(13):4722-31.

37. Fendt SM, Bell EL, Keibler MA, Davidson SM, Wirth GJ, Fiske B, et al. Metformin decreases glucose oxidation and increases the dependency of prostate cancer cells on reductive glutamine metabolism. Cancer Res. 2013;73(14):4429-38
38. Elhammali A, Ippolito JE, Collins L, Crowley J, Marasa J, Piwnica-Worms D. A high-throughput fluorimetric assay for 2-hydroxyglutarate identifies zaprinast as a glutaminase inhibitor. Can Dis. 2014;4(7):828-39.

39. Moore Z, Chakrabarti G, Luo X, Ali A, Hu Z, Fattah FJ, et al. NAMPT inhibition sensitizes pancreatic adenocarcinoma cells to tumor-selective, PAR-independent metabolic catastrophe and cell death induced by beta-lapachone. Cell Death Dis. 2015;6:e1599.

40. Qiu W, Su GH. Challenges and advances in mouse modeling for human pancreatic tumorigenesis and metastasis. Cancer Metastasis Rev. 2013;32(1-2):83-107

41. Hingorani SR, Wang L, Multani AS, Combs C, Deramaudt TB, Hruban RH, et al. Trp53R172H and KrasG12D cooperate to promote chromosomal instability and widely metastatic pancreatic ductal adenocarcinoma in mice. Cancer Cell. 2005;7(5):469-83

42. Savage RE, Hall T, Bresciano K, Bailey J, Starace M, Chan TC. Developmen and validation of a liquid chromatography-tandem mass spectrometry method for the determination of ARQ 501 (beta-lapachone) in plasma and tumors from nu/nu mouse xenografts. J Chromatogr B Analyt Technol Biomed Life Sci. 2008;872(1-2):148-53.

43. Pink JJ, Planchon SM, Tagliarino C, Varnes ME, Siegel D, Boothman DA. $\mathrm{NAD}(\mathrm{P}) \mathrm{H}$ :quinone oxidoreductase activity is the principal determinant of beta-lapachone cytotoxicity. J Biol Chem. 2000;275(8):5416-24

\section{Submit your next manuscript to BioMed Central and take full advantage of:}

- Convenient online submission

- Thorough peer review

- No space constraints or color figure charges

- Immediate publication on acceptance

- Inclusion in PubMed, CAS, Scopus and Google Scholar

- Research which is freely available for redistribution

Submit your manuscript at www.biomedcentral.com/submit 